\title{
Narrative review of the relationship between COVID-19 and PJP: does it represent coinfection or colonization?
}

\author{
Woon H. Chong ${ }^{1}$ (I) $\cdot$ Biplab K. Saha ${ }^{2} \cdot$ Amit Chopra $^{1}$
}

Received: 18 March 2021 / Accepted: 18 May 2021 / Published online: 31 May 2021

○) Springer-Verlag GmbH Germany, part of Springer Nature 2021

\begin{abstract}
Background Pneumocystis jirovecii (P. jirovecii) is increasingly identified on lower respiratory tract specimens of COVID19 patients. Our narrative review aims to determine whether the diagnosis of pneumocystis jirovecii pneumonia (PJP) in COVID-19 patients represents coinfection or colonization based on the evidence available in the literature. We also discuss the decision to treat COVID-19 patients with coinfection by PJP.

Methods A literature search was performed through the Pubmed and Web of Science databases from inception to March 10, 2021.

Results We identified 12 COVID-19 patients suspected to have PJP coinfection. All patients were critically ill and required mechanical ventilation. Many were immunosuppressed from HIV or long-term corticosteroids and other immunosuppressive agents. In both the HIV and non-HIV groups, severe lymphocytopenia was encountered with absolute lymphocyte and CD4+T cell count less than 900 and 200 cells/mm, respectively. The time to PJP diagnosis from the initial presentation was 7.8 (range 2-21) days. Serum lactate dehydrogenase and beta-D-glucan were elevated in those coinfected with PJP. All patients were treated with anti-PJP therapy, predominantly sulfamethoxazole-trimethoprim with corticosteroids. The overall mortality rate was $41.6 \%$, and comparable for both HIV and non-HIV groups.

Conclusion As the current evidence is restricted to case reports, the true incidence, risk factors, and prognosis of COVID-19 patients with PJP coinfections cannot be accurately determined. Comorbidities of poorly controlled HIV with lymphocytopenia and multiple immunosuppressive therapies are likely predisposing factors for PJP coinfection.
\end{abstract}

Keywords Severe acute respiratory syndrome coronavirus $2 \cdot$ SARS-CoV-2 $~$ Coronavirus disease 2019 · COVID-19 . Pneumocystis jirovecii pneumonia $\cdot$ Pneumocystis carinii pneumonia $\cdot$ PJP $\cdot$ PCP

\section{Introduction}

Since it was first recognized in December 2019, coronavirus disease 2019 (COVID-19) has led to the ongoing pandemic. While clinical care is highly focused on the diagnosis and management of COVID-19, there is emerging evidence that COVID-19 patients are at risk of developing concurrent pulmonary infections by bacterial and fungal microorganisms [1-3]. Moreover, during the pandemic, opportunistic

Woon H. Chong

chongw@amc.edu

1 Department of Pulmonary and Critical Care Medicine, Albany Medical Center, 43 New Scotland Avenue, Albany, NY, USA

2 Department of Pulmonary and Critical Care, Ozarks Medical Center, West Plains, MO, USA infections such as tuberculosis and Pneumocystis jirovecii pneumonia (PJP) have been increasingly described in COVID-19 patients, especially those with human immunodeficiency virus (HIV) [1, 4]. The difficulty in diagnosing PJP in patients with COVID-19 pneumonia is due to the many overlapping clinical, radiological, and laboratory abnormalities shared between these two often indistinguishable entities [5, 6]. PJP generally follows a more subacute course than COVID-19, particularly when considering milder form, with clinical manifestation of dry cough, dyspnea, and hypoxia [7-9]. PJP symptoms can last for several weeks before a diagnosis is made compared to COVID-19, with a median time to diagnosis of 7-9 days from illness onset $[10,11]$. The typical radiological features for COVID19 seen on CT imaging are diffuse, bilateral, peripheralpredominant multifocal lung infiltrates consisting of groundglass opacities (GGO); however, these findings can be seen 
in non-COVID-19-related pneumonia from viral, bacterial, and fungal etiologies $[5,6]$. The purpose of our narrative review is to determine whether PJP diagnosis in COVID-19 patients represents actual infection or colonization based on the evidence available in the literature.

\section{Methods}

We performed a literature search through the Pubmed and Web of Science databases from inception to March 10, 2021, using the search term in Medical Subjects Headings (MeSH) and title/abstract: ("coronavirus disease 2019" OR "COVID19" OR "COVID 19" OR "COVID19" OR "novel coronavirus" OR "2019 novel coronavirus" OR "2019-nCoV” OR "2019nCoV" OR "severe acute respiratory syndrome coronavirus 2" OR "SARS-CoV “ OR "SARS-CoV-2") AND ("Pneumocystis jirovecii pneumonia" OR "Pneumocystis carinii pneumonia" OR "Pneumocystis pneumonia" OR "Pneumocystis jirovecii" OR "Pneumocystis carinii" OR "pneumocystis" OR "PJP" OR "PCP"). No date restrictions were applied. We included studies: (1) involving hospitalized COVID-19 patients with PJP coinfections; (2) involving observational studies, case series, and case reports; and (3) with the diagnosis of COVID-19 infections made via RTPCR from a nasopharyngeal or oropharyngeal swab or bronchoalveolar lavage (BAL). We excluded: (1) other systematic reviews, literature reviews, editorials, and opinion articles, but references were screened for relevant articles; (2) studies discussing other infectious outbreaks; (3) studies on animals or in vitro studies; and (4) studies published in languages other than English were excluded if no translated version of the manuscript was available. We defined PJP coinfection in COVID-19 patients as those with Pneumocystis jirovecii (P. jirovecii) identification from lower respiratory tract (LRT) specimen such as sputum, endotracheal aspirate (ETA), and BAL by methods of positive microscopic staining (MS), immunofluorescence microscopy (IFM), or polymerase chain reaction (PCR) result and treated with anti-PJP therapy. LRT specimen can be obtained before or after the diagnosis of COVID-19 infection. Clinical features, including risk factors, radiological features, and laboratory findings, although supporting the diagnosis of PJP coinfection in COVID-19 patients, were not necessary but described in Tables 1 and 2. The following clinical characteristics were collected and summarized in Table 1 involving: age of the patient; gender; HIV status, absolute lymphocyte count (ALC) during PJP diagnosis; lowest CD4 + T cell count; long-term $(4$ weeks $<$ ) corticosteroid exposure before admission and other immunosuppressants; and radiological findings typical for PJP. In Table 2, the diagnosis, treatment, and outcomes were described: the highest respiratory support required, such as non-invasive mechanical ventilation
(NIMV) of bilevel positive airway pressure or continuous positive airway pressure, high-flow nasal cannula (HFNC), or invasive mechanical ventilation (IMV); days from symptoms onset to presentation; days from presentation to PJP diagnosis; LRT specimen source; serum lactate dehydrogenase (LDH) and beta-D-glucan (BDG) during PJP diagnosis; other microorganisms identified from respiratory source; and anti-PJP, antimicrobials, and anti-COVID-19 therapies received.

\section{Results}

\section{Clinical characteristics and lymphocyte count}

Twelve case reports describing PJP coinfection in COVID19 patients were identified (Fig. 1). The mean age of patients was $49.9 \pm 20.8$ years (Table 1 ). Out of the 12 patients included with COVID-19 and PJP coinfection, 58.3\% (7/12) had positive HIV status, and 41.7\% (5/12) had negative HIV status. All patients in the HIV group were male, but only $40 \%(2 / 5)$ were male in the non-HIV group. The common radiological features described on chest imaging suggestive of PJP in one-half of cases were GGO predominantly in the upper lobes of the lung, multiple lung cysts, and pneumothoraces. All COVID-19 patients were critically ill and required ICU admission during their respective hospitalization. For the highest level of respiratory support, 75\% (9/12) of patients required IMV, and the remainder required NIMV and HFNC (Table 2).

91.7\% (11/12) of COVID-19 patients were immunocompromised at baseline from either a positive HIV status or long-term exposure to immunosuppressants such as highdose corticosteroids, leflunomide, tacrolimus, mycophenolate mofetil (MMF), fludarabine, cyclophosphamide, and rituximab in the non-HIV group. These immunosuppressive therapies were for comorbidities of renal transplant, chronic lymphocytic leukemia (CLL), and connective tissue disease of rheumatoid arthritis (RA) and dermatomyositis. The overall ALC during PJP diagnosis was 520 cells $/ \mathrm{mm}^{3}$ (496 cells $/ \mathrm{mm}^{3}$ for the non-HIV group and 544 cells $/ \mathrm{mm}^{3}$ for HIV group), and the overall nadir CD4 + T cell counts were 96 cells $/ \mathrm{mm}^{3}$ (163 cells $/ \mathrm{mm}^{3}$ for the non-HIV group and 77 cells $/ \mathrm{mm}^{3}$ for HIV group).

\section{Diagnosis, outcome, and treatment}

The time to presentation from illness onset and time to PJP diagnosis from the initial presentation was highly variable, with a mean of 15.0 (range 1-28) days and 7.8 (range 2-21) days, respectively. COVID-19 patients with HIV had a delay in presentation from illness onset (mean 16.8 days vs. 13.2 days) than the non-HIV group (Table 2). No difference 


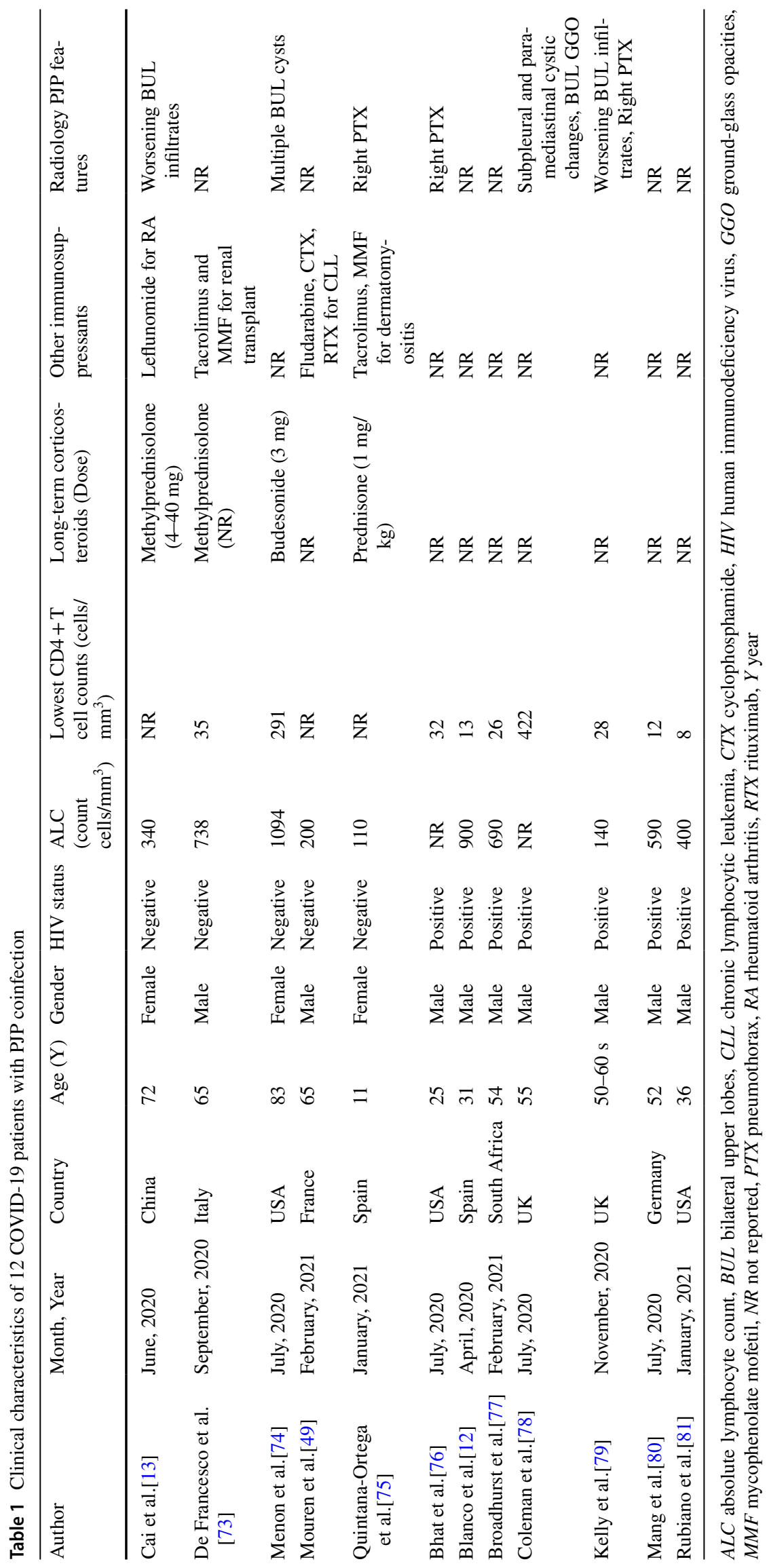




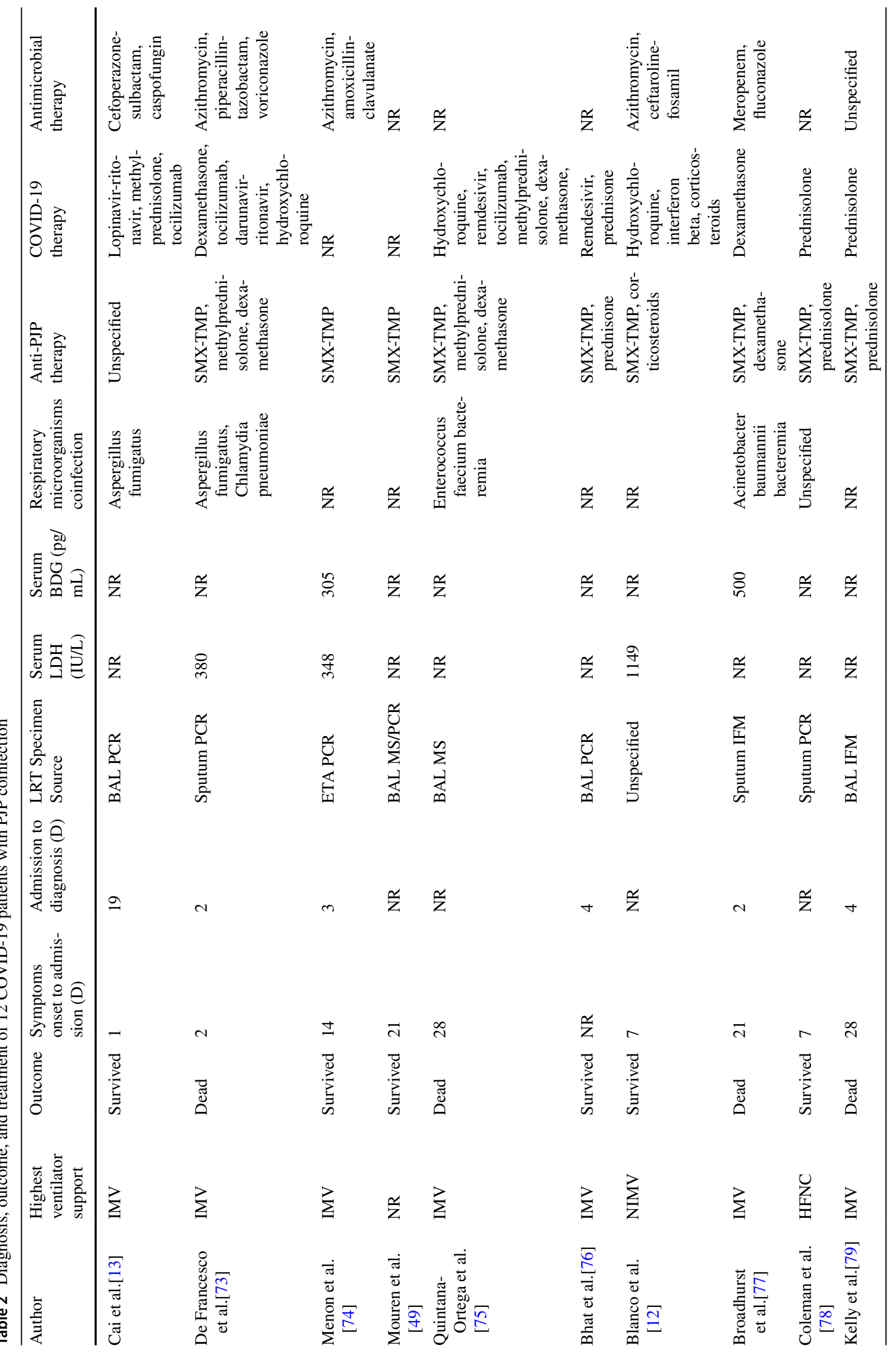




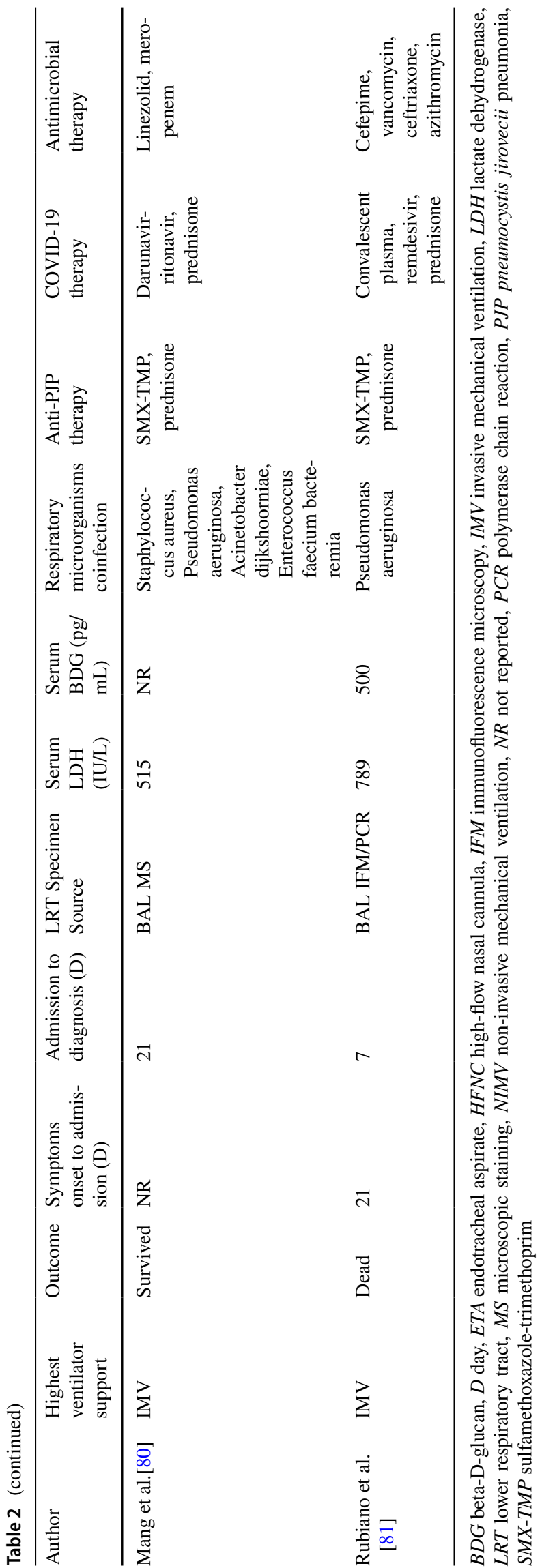

was observed in the duration of PJP diagnosis from presentation (mean 7.6 days vs. 8.0 days) in the HIV and non-HIV groups. The diagnosis of PJP coinfection was made by the LRT specimen source of BAL in $58.3 \%$ (7/12) and sputum in $25 \%$ (3/12) of COVID-19 patients (Table 2). In most cases, PCR [58.3\% (7/12)] was used to detect $P$. jirovecii, followed by MS and IFM [25\% (3/12)], respectively. Only one case report by Blanco et al. did not describe the source of the LRT specimen [12]. Serum LDH was measured in $41.7 \%$ (5/12) of COVID-19 patients, with elevated mean levels of $636 \mathrm{IU} / \mathrm{L}$. Serum BDG levels were only elevated in $25.0 \%$ (3/12) of COVID-19 patients, with a mean of $435 \mathrm{IU} / \mathrm{L}$. $41.6 \%$ (5/12) of patients with PJP and COVID-19 coinfection had other bacterial or fungal microorganisms identified from LRT specimens that suggested additional bacterial or fungal coinfections. The microorganisms isolated were Chlamydia pneumoniae, Staphylococcus aureus, Pseudomonas aeruginosa, Acinetobacter dijkshoorniae, and Aspergillus fumigatus. $25.0 \%$ (3/12) patients had underlying bacteremia from Acinetobacter baumannii and Enterococcus faecium.

The overall mortality rate was $41.6 \%(5 / 12)$, involving $40 \%(2 / 5)$ in the non-HIV group and $42.9 \%$ (3/7) in the HIV group (Table 2). 57.1\% (4/7) of patients with suspected bacterial or fungal coinfections died during hospitalization. Most patients [91.6\% (11/12)] with COVID-19 and PJP coinfections received anti-PJP therapy of sulfamethoxazole-trimethoprim (SMX-TMP) together with corticosteroids of dexamethasone, methylprednisolone, prednisolone, or prednisone. Cai et al. was the only case report that did not describe the type of anti-PJP therapy administered [13]. 83.3\% (10/12) of case reports described a variety of COVID19 therapies given, which includes anti-viral (e.g., lopinavir-ritonavir, darunavir-ritonavir, and remdesivir), immunomodulators (e.g., corticosteroids, hydroxychloroquine, interferon beta, and tocilizumab), and convalescent plasma. $66.7 \%(8 / 12)$ of patients received antimicrobial therapy of antibiotics and/or antifungals during hospitalization.

\section{Discussion}

We identified 12 COVID-19 patients to have coinfections by PJP in which more than one-half of patients had a positive HIV status. Common radiological features suggestive of PJP were GGO predominantly in the upper lobes of the lung, multiple lung cysts, and pneumothoraces. All patients were critically ill and required IMV. Many patients were immunosuppressed from HIV or long-term corticosteroids and other immunosuppressive agents. In both the HIV and non-HIV group, severe lymphocytopenia $(<1000$ cells/ $\mathrm{mm}^{3}$ ) was encountered with ALC, and CD4 + T cell count was less than 900 and 200 cells $/ \mathrm{mm}^{3}$, respectively. The time to presentation from illness onset was 15.0 (range 1-28) 




Fig. 1 Flowchart of included case reports

days, and the time to PJP diagnosis from the initial presentation was 7.8 (range 2-21) days. Patients with HIV had a delay in presentation from illness onset than the non-HIV group. PJP diagnosis was made by LRT specimen source of BAL in more than one-half of patients, and either ETA or sputum in the remaining patients. PCR was frequently used to examine the LRT specimen for $P$. jirovecii over MS and IFM. Although serum LDH and BDG were elevated during PJP diagnosis, less than 50\% of COVID-19 patients had serum LDH and BDG examined. All patients were treated with anti-PJP therapy, predominantly SMX-TMP with corticosteroids. The majority of patients received COVID-19 therapies of anti-viral, immunomodulators, and convalescent plasma. Nevertheless, the overall mortality rate was $41.6 \%$ and appeared similar for both HIV and non-HIV groups. $58.3 \%$ of patients were suspected to be coinfected by other bacterial and fungal microorganisms, in which more than half of these patients died during hospitalization despite antimicrobial therapies.

In contrast, concurrent PJP infection has not been reported in patients with severe acute respiratory syndrome (SARS) and Middle East respiratory syndrome (MERS). In a 2019 study, up to 7\% of critically ill influenza A patients had positive $P$. jirovecii in their BAL PCR. However, the authors did not comprehensively delineate whether those patients had actual infection versus colonization [14]. Several cases have been reported in the literature involving both immunocompetent and immunocompromised HIV-negative status influenza A patients with confirmed PJP demonstrating an absolute lymphocyte count and CD4 + T cell count less than 600 cells $/ \mathrm{mm}^{3}$ and 200 cells $/ \mathrm{mm}^{3}$, respectively [15-17]. Influenza A is known to deplete both the innate alveolar immunity involving dendritic cells, macrophages, and monocytes together with $\mathrm{T}$ and $\mathrm{B}$ cells as a result of influenza A-induced cell apoptosis and migration of these cells from the circulation to the infected respiratory tract as a consequence of infection [18-21]. Lymphocytopenia seen in up to $69 \%$ of influenza A patients typically recover after 1-3 weeks from illness onset or even after the fever subsides [22, 23].

\section{Factors favoring PJP coinfection}

Lung infiltrates of GGOs predominantly in the bilateral upper lobes are useful distinguishing radiological features for PJP [5, 24]. However, when PJP is superimposed on 
COVID-19 pneumonia, these findings are indistinguishable between the two entities, mainly when lung infiltrates are diffuse. The involvement of lung typically more diffuse in those without HIV than those with HIV ( $86 \%$ vs. $44 \% ; P=0.02)$, which further complicates the diagnosis of PJP coinfection in advanced COVID-19 pneumonia [9, 25-28]. Cavitation, pleural effusions, and lymphadenopathies are rarely seen in COVID-19 pneumonia and PJP; however, these radiological findings may support a diagnosis of superimposed bacterial and fungal pneumonia [5, 6]. PJP coinfection should be part of the differential diagnosis when multiple lung cysts are observed, possibly with spontaneous pneumothorax in the setting of immunocompromised status [6,28]. These cysts, typically diffuse, bilateral, and thin-walled, are seen in up to $56 \%$ of PJP patients and may resolve with PJP treatment. However, the frequency of cystic lesions can be less than $10 \%$ in patients without concurrent HIV infection [28, 29]. The occurrence of pneumothorax is similar for PJP patients, regardless of HIV-positivity status [5, 6, 28, 30]. Compared to COVID-19 pneumonia, pneumothorax is a rare radiological finding and observed in less than $5 \%$ of cases [27, 31].

In the absence of classical risk factors of HIV, PJP coinfection is increasingly identified in COVID-19 patients with lymphocytopenia and multiple immunosuppressants. Lymphocytopenia, observed in up to $83 \%$ of hospitalized COVID-19 patients, is one of the many etiologies predisposing to concurrent PJP infections, a pathogen commonly seen in patients with defects in T cell immunity [8]. The innate and adaptive immune system is activated during COVID-19 infection, causing the release of cytokines to trigger the recruitment of macrophages, monocytes, and both $\mathrm{T}$ and $\mathrm{B}$ cells from the circulation towards the inflamed tissues [32, 33]. As a result of the proinflammatory cytokines' release and COVID-19-induced lymphocyte apoptosis, the immunosuppression stage follows, characterized by sustained lymphocytopenia that occurs within a week from COVID-19 illness onset before returning to normal levels, two weeks later [34, 35]. An ALC less than 1700 cell $/ \mathrm{mm}^{3}$ had $84 \%$ sensitivity and $55 \%$ specificity for predicting a CD4 $+\mathrm{T}$ cell count of less than $200 \mathrm{cell} / \mathrm{mm}^{3}$.[36] Unlike ALC, CD4 + T cell count is rarely measure in COVID-19 patients unless a strong suspicion exists for concurrent HIV infection or a severely immunocompromised state. Patients diagnosed with PJP had CD4 + T cell count of less than 100 cell/ $\mathrm{mm}^{3}$ in $78.7 \%$ of cases, followed by $16.2 \%$ and $5.1 \%$ when CD4 + T cell count was $100-200$ cell $/ \mathrm{mm}^{3}$, and 200 cell/ $\mathrm{mm}^{3}$ and above, respectively [37]. However, this datum was extracted from an observational study involving HIVpatients. Therefore, strong suspicion should exist for PJP coinfection in those with severe lymphocytopenia, regardless of HIV status. Second, COVID-19-induced alveolar macrophage dysfunction is likely responsible for $P$. jirovecii evading phagocytosis by the alveolar macrophage, primary host defense while dysregulating surfactant production [38-40]. Surfactant is essential to facilitate attachment of $P$. jirovecii to the macrophages for phagocytosis and inhibit replication that explains why $P$. jirovecii favors the upper lobe of the lungs containing the least amount of surfactant required to maintain lung compliance.

Corticosteroids have anti-inflammatory and immunomodulatory properties by inhibiting cytokines, decreasing leukocyte migration, and triggering lymphocytes apoptosis, specifically T cells $[33,35]$. Therefore, corticosteroids help prevent and treat cytokine storm and ARDS in COVID-19 patients. A prolonged course (4 weeks $<$ ) of corticosteroid with a median daily dose equivalent to $30 \mathrm{mg}$ of prednisone is often associated with PJP infection [41, 42]. However, patients receiving does as low as $15 \mathrm{mg}$ or have baseline lymphocytopenia (HR 10.7; 95\% CI 1.8-63.8; $P=0.01)$ are also at increased risk [41]. CLL patients are already immunocompromised due to the exhaustion of CD4 $+\mathrm{T}$ cell and immunoglobulins production, an essential component of adaptive immunity towards the advanced stage of the disease. The addition of other immunosuppressants such as fludarabine and rituximab will induce a profound CD4 $+\mathrm{T}$ cell lymphocytopenia and hypogammaglobulinemia predisposing to opportunistic infections such as PJP [43]. Tocilizumab, an IL-6 inhibitor biologic, is commonly used in COVID-19 and RA patients, with reported PJP events of 0.28 per 100 patient-years [44, 45]. MMF and tacrolimus have been shown to deplete lymphocytes, specifically $\mathrm{T}$ cells and complement levels, increasing the risk of opportunistic infection such as PJP when used in conjunction with corticosteroids [46, 47].

Despite a huge variation in timing from illness onset to presentation in our study population, HIV-positive patients had a delay in clinical presentation from illness onset than those without HIV (Table 2). This is frequently seen in many HIV patients with PJP coinfection, in which the HIV group had a longer duration of illness (18 days vs. 10 days; $P<0.05$ ) than the non-HIV group [30]. BAL cell differentials are often non-specific in patients with COVID-19 pneumonia; however, BAL cell counts that are predominant lymphocytosis $(13 \%<)$ with flow cytometry showing high levels of CD8 $+\mathrm{T}$ cells $(30 \%<)$ and cell viability $(80 \%<$ ) but low CD $4+\mathrm{T}$ cells $(50 \%>)$ and CD $4+/$ $\mathrm{CD} 8+\mathrm{T}$ cell ratio $(2.5>)$ are suggestive of PJP coinfection [48]. Mouren et al. was the only case report reporting BAL findings in COVID-19 patients with PJP coinfection, demonstrating a total white cell count of $468 \times 10^{3}$ cells $/ \mathrm{mm}^{3}$ and 38\% lymphocytes [49]. Nevertheless, the significance of low levels of PJP detected by PCR and BAL cell count differentials need to be interpreted in conjunction with clinical context. 


\section{Factors favoring colonization}

Our study results demonstrated that all patients in the HIV group were male, but only $40 \%$ were males in the non-HIV group (Table 1). Furthermore, the mortality rate was similar for both HIV and non-HIV groups (Table 2). This is inconsistent with the findings from several observational studies demonstrating that COVID-19 patients without HIV are predominantly males and have a higher fatality rate than COVID-19 patients with HIV. In 2008, an observational study comparing 72 PJP patients revealed that the non-HIV group were males ( $80.4 \%$ vs. $40.7 \% ; P<0.05)$, had a higher severity of illness ( $38 \%$ vs $27 \% ; P<0.05)$, and greater ICUmortality ( $48 \%$ vs. $17 \% ; P<0.05)$ [30]. In 2020, a large observational study involving 3938 PJP patients demonstrated that a greater proportion of non-HIV patients were males (79.85 vs. $48.5 \% ; P<0.01)$, more likely symptomatic $(38.2 \%$ vs. $30.3 \% ; P<0.01)$, and had a higher mortality (6.6\% vs. $4.2 \% ; P<0.01)$ than those with HIV [50]. The difference in host inflammatory immune response rather than virulence of the $P$. jirovecii explains the acute illness, severe hypoxia, and rapid decline in respiratory status in non-HIV patients [28, 29]. Given the evidence of proinflammatory cytokines, innate and adaptive immune factors playing a significant role in promoting severe COVID-19, the immunosuppressive characteristics of HIV can lead to better outcomes in COVID-19 patients with PJP coinfections [32,33]. Critically ill COVID-19 patients will generally have CD4 + T cell response heavily skewed towards $\mathrm{T}$ helper cell type 2 (TH2) activation. TH2 is known to secrete interleukin-6, a cytokine predominantly responsible for COVID-19-induced immune dysregulation, but is suppressed in those with HIV $[51,52]$. Therefore, the findings above could suggest colonization instead of actual infection. An observational study by Alanio et al. reported that in 108 non-HIV COVID-19 patients requiring IMV, the detection of P. jirovecii by PCR obtained by routine bronchoscopy with BAL was 9.3\% [53]. More than one-half of these patients had low serum BDG and were not treated for PJP, where outcomes, including mortality, did not differ, suggesting possible colonization.

\section{Decision on treatment}

The gold-standard staining or immunofluorescence microscopy to detect $P$. jirovecii in LRT specimens, frequently obtained from BAL, are used to confirm PJP [9]. Like many patients without HIV, the diagnosis of PJP becomes challenging, in whom the burden of PJP is generally lower and limits the sensitivity of microscopy [9]. Given the high sensitivity of $P$. jirovecii PCR in detecting low fungal loads, even in non-HIV patients, the distinction between actual infection and colonization is a common problem [54, 55]. PCR has $87.2 \%$ sensitivity and $92.2 \%$ specificity; $51.5 \%$ positive predictive value and $98.7 \%$ negative predictive value for diagnosing PJP [56]. Therefore, a negative PCR can exclude PJP and allows cessation of therapy. Studies have suggested using quantitative $P$. jirovecii PCR and serum BDG to distinguish between actual infection and colonization $[57,58]$. Specifically, a quantitative BAL PCR greater than $1.6 \times 10^{3}$ DNA copies/ $\mathrm{uL}$ and BDG cut-off level of $100 \mathrm{pg} / \mathrm{mL}$ can distinguish between $P$. jirovecii infection and colonization with close to $100 \%$ sensitivity, regardless of HIV status [57]. In combination, the high sensitivity of PJP PCR from BAL and serum BDG may even prevent the need for IFM. It has even been proposed that in symptomatic non-HIV patients with positive serum BDG and PCR but negative sputum or BAL microscopy, clinical suspicion should remain high for PJP [9]. Nevertheless, these resources were lacking in many studies included (Table 2).

In critically ill COVID-19 patients who are unable to tolerate diagnostic bronchoscopy, compatible clinical and radiological features with elevated serum biomarkers levels of LDH and BDG may be the only useful tools to warrant an empirical trial of anti-PJP therapy such as sulfamethoxazole-trimethoprim (SMX-TMP) [59]. BDG levels are typically low, less than the cut-off of $80 \mathrm{pg} / \mathrm{mL}$ in COVID-19 infection, as the COVID-19 virus lacks the polysaccharide cell walls present in certain fungal and bacteria microorganisms [60, 61]. In 2011, a meta-analysis demonstrated that serum BDG had $94.8 \%$ sensitivity and $86.3 \%$ specificity for the diagnosis of PJP in the setting of compatible clinical manifestations and risk factors [62]. LDH levels are commonly elevated in both COVID-19 and PJP patients, making it a sensitive but not specific biomarker to distinguish between the two entities [8, 63]. However, serum LDH has been shown higher among non-survivors than survivors for both COVID-19 (mean $521 \mathrm{IU} / \mathrm{L}$ vs. $253 \mathrm{IU} / \mathrm{L} ; P<0.01$ ) and PJP (mean 447 IU/L vs. $340 \mathrm{IU} / \mathrm{L} ; P<0.05$ ) [63, 64]. An observational study suggested that serum LDH level greater than $450 \mathrm{IU} / \mathrm{L}$ has close to $100 \%$ sensitivity in diagnosing PJP in the context of compatible clinical symptoms in nonCOVID-19 patients [63].

When clinical suspicion for PJP is high, treatment can be initiated even before making a definitive diagnosis because $P$. jirovecii persists in respiratory specimens for up to 3 weeks after adequate treatment is initiated [65]. Clinical improvement with anti-PJP therapy can be expected around 4-8 days $[9,55]$. Serum BDG, despite being a reliable, adjunct diagnostic marker, may indicate favorable treatment response with decreasing levels but does not always reflect the severity and prognosis in PJP patients [66-68]. With anti-PJP therapy, serum BDG will generally reduce by a median of 17 (IQR 0-82) pg/mL over a week and may occasionally increase in levels for a few weeks before declining, and does not reflect treatment failure [66, 67]. 


\section{Limitations}

The major limitation of our narrative review is the literature on PJP coinfection in COVID-19 patients is limited to 12 case reports. Therefore, the true incidence, risk factors, and prognosis of COVID-19 patients with concurrent PJP infections cannot be precisely determined. The difficulties in confirming the diagnosis of PJP are due to the concerns of viral transmission between COVID-19 patients and healthcare workers, and worsening hemodynamic status in a severely hypoxemic COVID-19 patient while performing aerosolized generating procedures such as bronchoscopy to obtain LRT specimen of sufficient quality. Therefore, bronchoscopy is often discouraged unless an alternate diagnosis provided by BAL would significantly impact clinical management [69]. However, recent observational studies have demonstrated the low risk for viral transmission to healthcare workers when adhering to WHO guidelines on airborne precautions for aerosolgenerating procedures [70-72]. Other important diagnostic and prognostic markers for PJP, such as serum BDG and the ratio of partial pressure of arterial oxygen to fractional inspired oxygen ( $\mathrm{paO} 2 / \mathrm{FiO} 2)$, were not consistently evaluated. The overall mortality rate could have been affected by other microorganisms causing secondary pulmonary infections or sepsis among the $58.3 \%$ of patients included in our study, in which more than half of these patients died during hospitalization despite antimicrobial therapies. However, it is even possible that these bacterial and fungal microorganisms identified could be colonizers similar to P. jirovecii.

\section{Conclusion}

There is huge variability in timing from illness onset to presentation, and presentation to PJP diagnosis in COVID19 patients, regardless of HIV status. It remains unclear if COVID-19 patients with $P$. jirovecii identified on LRT specimens represent actual infection versus colonization due to the similarities in clinical presentation. Comorbidities of poorly controlled HIV with lymphocytopenia and multiple immunosuppressive therapies are likely predisposing factors for PJP coinfection. As PJP coinfection is under-recognized and under-reported, causing the lack of diagnostic testing, the true incidence and outcomes of COVID-19 patients with PJP coinfection remain uncertain. Incorporating HIV screening of all patients admitted for COVID-19 into institutional protocols is a sensible approach to reducing diagnostic uncertainty and anchoring biases due to significant overlap in clinical presentation.
It essential to maintain a broad differential diagnosis and prudent to consider additional diagnostic testing for $P$. jirovecii in COVID-19 patients, mainly when there is a lack of clinical improvement in respiratory status, radiological features of lung cysts and possibly with pneumothorax, and laboratory findings of elevated serum LDH and BDG, even in the absence of classical risk factors such as HIV. As the current evidence in the literature is restricted to case reports, the incidence, risk factors, and prognosis of COVID-19 patients with PJP coinfections cannot be accurately determined. A large, well-designed study is needed to differentiate between PJP coinfections and colonization, possibly with the development of sensitive diagnostic criteria due to the similarities in clinical presentation.

Author contributions All authors had access to the data and were involved in writing the manuscript.

\section{References}

1. Chong WH, Saha BK, Ananthakrishnan Ramani, Chopra A. Stateof-the-art review of secondary pulmonary infections in patients with COVID-19 pneumonia. Infection. 2021 Mar 11:1-15. https:// doi.org/10.1007/s15010-021-01602-z.

2. Chong WH, Neu KP. The incidence, diagnosis, and outcomes of COVID-19-associated pulmonary aspergillosis (CAPA): a systematic review. J Hosp Infect. 2021. https://doi.org/10.1007/ s15010-021-01602-z.

3. Chong WH, Chieng H, Tiwari A, Beegle S, Feustel PJ, Ghalib $\mathrm{S}$, et al. Incidence and risk factors for secondary pulmonary infections in patients hospitalized with coronavirus disease 2019 pneumonia. Am J Med Sci. 2021. https://doi.org/10.1016/j.amjms. 2021.04.007.

4. Saunders MJ, Evans CA. COVID-19, tuberculosis and poverty: preventing a perfect storm. Eur Respir J. 2020;56:2001348.

5. Duzgun SA, Durhan G, Demirkazik FB, Akpinar MG, Ariyurek OM. COVID-19 pneumonia: the great radiological mimicker. Insights Imaging. 2020;11:118.

6. Parekh M, Donuru A, Balasubramanya R, Kapur S. Review of the chest CT differential diagnosis of ground-glass opacities in the COVID Era. Radiology. 2020;297:E289-302.

7. Rodriguez-Morales AJ, Cardona-Ospina JA, Gutiérrez-Ocampo E, Villamizar-Peña R, Holguin-Rivera Y, Escalera-Antezana JP, et al. Clinical, laboratory and imaging features of COVID-19: a systematic review and meta-analysis. Travel Med Infect Dis. 2020. https://doi.org/10.1056/NEJMoa2002032.

8. Guan W-J, Ni Z-Y, Hu Y, Liang W-H, Ou C-Q, He J-X, et al. Clinical characteristics of coronavirus disease 2019 in China. $\mathrm{N}$ Engl J Med. 2020;382:1708-20.

9. White PL, Price JS, Backx M. Therapy and management of Pneumocystis jirovecii infection. J Fungi Basel Switz. 2018. https://doi. org/10.3390/jof4040127.

10. Wang D, Hu B, Hu C, Zhu F, Liu X, Zhang J, et al. Clinical characteristics of 138 hospitalized patients with 2019 novel coronavirus-infected pneumonia in Wuhan China. JAMA. 2020. https:// doi.org/10.1001/jama.2020.1585.

11. Huang C, Wang Y, Li X, Ren L, Zhao J, Hu Y, et al. Clinical features of patients infected with 2019 novel coronavirus in Wuhan China. Lancet. 2020;395:497-506. 
12. Blanco JL, Ambrosioni J, Garcia F, Martínez E, Soriano A, Mallolas J, et al. COVID-19 in patients with HIV: clinical case series. Lancet HIV. 2020;7:e314-6.

13. Cai S, Sun W, Li M, Dong L. A complex COVID-19 case with rheumatoid arthritis treated with tocilizumab. Clin Rheumatol. 2020;39:2797-802.

14. Beumer MC, Koch RM, van Beuningen D, OudeLashof AM, van de Veerdonk FL, Kolwijck E, et al. Influenza virus and factors that are associated with ICU admission, pulmonary co-infections and ICU mortality. J Crit Care. 2019;50:59-65.

15. Burke J, Soubani AO. Influenza and Pneumocystis jirovecii pneumonia in an allogeneic hematopoietic stem cell transplantation recipient: coinfection or superinfection? Transpl Infect Dis. 2018. https://doi.org/10.1111/tid.12802.

16. Franconi I, Monari C, Tutone M, et al. Pneumocystosis as a Complication of H1N1 Influenza A Infection in an HIV-Positive Patient on Effective cART. Open Forum Infect Dis. 2019;6(4):ofz105. https://doi.org/10.1093/ofid/ofz105.

17. Marwah V, Singh S, Govind R, Shergill S, Vasan A. Pneumocystis jiroveci pneumonia and H1N1 co-infection in an immunocompetent patient. Indian J Chest Dis Allied Sci. 2019;61:219-220.

18. Mao H, Liu Y, Sia SF, Peiris JSM, Lau Y-L, Tu W. Avian influenza virus directly infects human natural killer cells and inhibits cell activity. Virol Sin. 2017;32:122-9.

19. Wang X, Tan J, Biswas S, Zhao J, Devadas K, Ye Z, et al. Pandemic influenza $A$ (H1N1) virus infection increases apoptosis and HIV-1 replication in HIV-1 infected jurkat cells. Viruses. 2016. https://doi.org/10.3390/v8020033.

20. Lewis DE, Gilbert BE, Knight V. Influenza virus infection induces functional alterations in peripheral blood lymphocytes. J Immunol Baltim Md 1950. 1986;137:3777-81.

21. Nichols JE, Niles JA, Roberts NJ. Human lymphocyte apoptosis after exposure to influenza A virus. J Virol. 2001;75:5921-9.

22. Cao B, Li X-W, Mao Y, Wang J, Lu H-Z, Chen Y-S, et al. Clinical features of the initial cases of 2009 pandemic influenza A (H1N1) virus infection in China. N Engl J Med. 2009:361:2507-17.

23. Cheng Y, Zhao H, Song P, Zhang Z, Chen J, Zhou Y-H. Dynamic changes of lymphocyte counts in adult patients with severe pandemic H1N1 influenza A. J Infect Public Health. 2019;12:878-83.

24. Chong WH, Saha BK, Austin A, Chopra A. The Significance of Subpleural Sparing in CT Chest: A State-of-the-Art Review. Am J Med Sci. 2021 Apr;361(4):427-435. https://doi.org/10.1016/j. amjms.2021.01.008.

25. Pan F, Ye T, Sun P, Gui S, Liang B, Li L, Zheng D, Wang J, Hesketh RL, Yang L, Zheng C. Time Course of Lung Changes at Chest CT during Recovery from Coronavirus Disease 2019 (COVID-19). Radiology. 2020 Jun;295(3):715-721. https://doi. org/10.1148/radiol.2020200370.

26. Bernheim A, Mei X, Huang M, Yang Y, Fayad ZA, Zhang N, Diao K, Lin B, Zhu X, Li K, Li S, Shan H, Jacobi A, Chung M. Chest CT Findings in Coronavirus Disease-19 (COVID-19): Relationship to Duration of Infection. Radiology. 2020;295(3):200463. https://doi.org/10.1148/radiol.2020200463.

27. Ding X, Xu J, Zhou J, Long Q. Chest CT findings of COVID19 pneumonia by duration of symptoms. Eur J Radiol. 2020;127:109009.

28. Kanne JP, Yandow DR, Meyer CA. Pneumocystis jiroveci pneumonia: high-resolution CT findings in patients with and without HIV infection. Am J Roentgenol. 2012;198:W555-61.

29. Hardak E, Brook O, Yigla M. Radiological features of Pneumocystis jirovecii pneumonia in immunocompromised patients with and without AIDS. Lung. 2010;188:159-63.

30. Monnet X, Vidal-Petiot E, Osman D, Hamzaoui O, Durrbach A, Goujard C, et al. Critical care management and outcome of severe Pneumocystis pneumonia in patients with and without HIV infection. Crit Care. 2008;12:R28.
31. Chong WH, Saha BK, Hu K, Chopra A. The incidence, clinical characteristics, and outcomes of pneumothorax in hospitalized COVID-19 patients: a systematic review. Heart Lung. 2021. https://doi.org/10.1016/j.hrtlng.2021.04.005.

32. Qin C, Zhou L, Hu Z, Zhang S, Yang S, Tao Y, et al. Dysregulation of immune response in patients with coronavirus 2019 (COVID-19) in Wuhan China. Clin Infect Dis. 2020;71:762-8.

33. Li H, Liu L, Zhang D, Xu J, Dai H, Tang N, et al. SARS-CoV-2 and viral sepsis: observations and hypotheses. The Lancet. 2020;395:1517-20.

34. Liu J, Li S, Liu J, Liang B, Wang X, Wang H, et al. Longitudinal characteristics of lymphocyte responses and cytokine profiles in the peripheral blood of SARS-CoV-2 infected patients. EBioMedicine. 2020;55:102763.

35. Lin L, Lu L, Cao W, Li T. Hypothesis for potential pathogenesis of SARS-CoV-2 infection-a review of immune changes in patients with viral pneumonia. Emerg Microbes Infect. 2020;9:727-32.

36. Napoli AM, Maughan B, Murray R, Maloy K, Milzman D. Use of the relationship between absolute lymphocyte count and cd4 count to improve earlier consideration of pneumocystis pneumonia in HIV-positive emergency department patients with pneumonia. J Emerg Med. 2013;4:28-35.

37. Stansell JD, Osmond DH, Charlebois E, LaVange L, Wallace JM, Alexander BV, et al. Predictors of Pneumocystis carinii pneumonia in HIV-infected persons. Pulmonary complications of HIV infection study group. Am J Respir Crit Care Med. 1997;155:60-6.

38. PrÃ@vost M-C, Escamilla R, Aliouat EM, CerÃ@ N, Coudert P, Dei-Cas EXV. Pneumocystosis pathophysiology. FEMS Immunol Med Microbiol. 1998;22:123-8.

39. Dalskov L, Møhlenberg M, Thyrsted J, Blay-Cadanet J, Poulsen ET, Folkersen BH, et al. SARS-CoV-2 evades immune detection in alveolar macrophages. EMBO Rep. 2020;21:e51252.

40. Wang C, Xie J, Zhao L, Fei X, Zhang H, Tan Y, et al. Alveolar macrophage dysfunction and cytokine storm in the pathogenesis of two severe COVID-19 patients. EBioMedicine. 2020;57:102833.

41. Park JW, Curtis JR, Kim MJ, Lee H, Song YW, Lee EB. Pneumocystis pneumonia in patients with rheumatic diseases receiving prolonged, non-high-dose steroids-clinical implication of primary prophylaxis using trimethoprim-sulfamethoxazole. Arthritis Res Ther. 2019;21:207.

42. Yale SH, Limper AH. Pneumocystis carinii pneumonia in patients without acquired immunodeficiency syndrome: associated illness and prior corticosteroid therapy. Mayo Clin Proc. 1996;71:5-13.

43. Forconi F, Moss P. Perturbation of the normal immune system in patients with CLL. Blood. 2015;126:573-81.

44. Koike T, Harigai M, Inokuma S, Ishiguro N, Ryu J, Takeuchi T, et al. Postmarketing surveillance of tocilizumab for rheumatoid arthritis in Japan: interim analysis of 3881 patients. Ann Rheum Dis. 2011;70:2148-51.

45. Gupta S, Wang W, Hayek SS, Chan L, Mathews KS, Melamed ML, et al. Association between early treatment with tocilizumab and mortality among critically ill patients with COVID-19. JAMA Intern Med. 2021;181:41-51.

46. Duncan MD. Transplant-related immunosuppression: a review of immunosuppression and pulmonary infections. Proc Am Thorac Soc. 2005;2:449-55.

47. Merayo-Chalico J, Gomez-Martin D, Pineirua-Menendez A, Santana-De Anda K, Alcocer-Varela J. Lymphopenia as risk factor for development of severe infections in patients with systemic lupus erythematosus: a case-control study. QJM. 2013;106:451-7.

48. Vedder V, Schildgen V, Lüsebrink J, Tillmann RL, Domscheit B, Windisch W, et al. Differential cytology profiles in bronchoalveolar lavage (BAL) in COVID-19 patients: a descriptive observation and comparison with other corona viruses, Influenza virus, 
Haemophilus influenzae, and Pneumocystis jirovecii. Medicine (Baltimore). 2021;100:e24256.

49. Mouren D, Goyard C, Catherinot E, Givel C, Chabrol A, Tcherakian C, et al. COVID-19 and Pneumocystis jirovecii pneumonia: back to the basics. Respir Med Res. 2021;79:100814.

50. Gold JAW, Jackson BR, Benedict K. Possible diagnostic delays and missed prevention opportunities in pneumocystis pneumonia patients without hiv: analysis of commercial insurance claims data-United States, 2011-2015. Open Forum Infect Dis. 2020;7:ofaa255.

51. Roncati L, Nasillo V, Lusenti B, Riva G. Signals of Th2 immune response from COVID-19 patients requiring intensive care. Ann Hematol. 2020;99:1419-20.

52. Herold T, Jurinovic V, Arnreich C, Lipworth BJ, Hellmuth JC, von Bergwelt-Baildon M, et al. Elevated levels of IL-6 and CRP predict the need for mechanical ventilation in COVID-19. J Allergy Clin Immunol. 2020;146:128-136.e4.

53. Alanio A, Dellière S, Voicu S, Bretagne S, Mégarbane B. The presence of Pneumocystis jirovecii in critically ill patients with COVID-19. J Infect. 2020. https://doi.org/10.1016/j.jinf.2020.10. 034 .

54. Mühlethaler K, Bögli-Stuber K, Wasmer S, von Garnier C, Dumont P, Rauch A, et al. Quantitative PCR to diagnose Pneumocystis pneumonia in immunocompromised non-HIV patients. Eur Respir J. 2012;39:971-8.

55. Blaize M, Mayaux J, Luyt C-E, Lampros A, Fekkar A. COVID19-related respiratory failure and lymphopenia do not seem associated with pneumocystosis. Am J Respir Crit Care Med. 2020;202:1734-6.

56. Azoulay É, Bergeron A, Chevret S, Bele N, Schlemmer B, Menotti $\mathrm{J}$. Polymerase chain reaction for diagnosing pneumocystis pneumonia in non-HIV immunocompromised patients with pulmonary infiltrates. Chest. 2009;135:655-61.

57. Damiani C, Le Gal S, Da Costa C, Virmaux M, Nevez G, Totet A. Combined quantification of pulmonary Pneumocystis jirovecii DNA and Serum (1->3)- -D-Glucan for differential diagnosis of pneumocystis pneumonia and pneumocystis colonization. J Clin Microbiol. 2013;51:3380-8.

58. Matsumura $\mathrm{Y}$, Ito $\mathrm{Y}$, Iinuma $\mathrm{Y}$, Yasuma $\mathrm{K}$, Yamamoto M, Matsushima A, et al. Quantitative real-time PCR and the $(1 \rightarrow$ $3)-\beta$-d-glucan assay for differentiation between Pneumocystis jirovecii pneumonia and colonization. Clin Microbiol Infect. 2012;18:591-7.

59. Barben J, Quipourt V, Vovelle J, Putot A, Manckoundia P. Not COVID-19, don't overlook pneumocystis in patients on Gefitinib! Curr Oncol. 2021;28:961-4.

60. White PL, Dhillon R, Cordey A, Hughes H, Faggian F, Soni S, et al. A national strategy to diagnose COVID-19 associated invasive fungal disease in the ICU. Clin Infect Dis. 2020. https://doi. org/10.2139/ssrn.3644400.

61. Lei Y, Song Y, Shu Y, Zhao Y, Huo X, Wang H, et al. Fungal antigenemia in patients with severe coronavirus disease 2019 (COVID-19): the facts and challenges. J Microbiol Immunol Infect Wei Mian Yu Gan Ran Za Zhi. 2020;53:657-9.

62. Karageorgopoulos DE, Qu J-M, Korbila IP, Zhu Y-G, Vasileiou VA, Falagas ME. Accuracy of $\beta$-D-glucan for the diagnosis of Pneumocystis jirovecii pneumonia: a meta-analysis. Clin Microbiol Infect. 2013;19(1):39-49.

63. Zaman MK, White DA. Serum lactate dehydrogenase levels and Pneumocystis carinii pneumonia. Diagnostic and prognostic significance. Am Rev Respir Dis. 1988;137:796-800.

64. Zhou F, Yu T, Du R, Fan G, Liu Y, Liu Z, et al. Clinical course and risk factors for mortality of adult inpatients with COVID19 in Wuhan, China: a retrospective cohort study. Lancet. 2020;395:1054-62.
65. Roger PM, Vandenbos F, Pugliese P, De Salvador F, Durant J, Le Fichoux Y, et al. Persistence of Pneumocystis carinii after effective treatment of $P$. carinii pneumonia is not related to relapse or survival among patients infected with human immunodeficiency virus. Clin Infect Dis. 1998;26:509-10.

66. Koga M, Koibuchi T, Kikuchi T, Nakamura H, Miura T, Iwamoto A, et al. Kinetics of serum $\beta$-D-glucan after pneumocystis pneumonia treatment in patients with AIDS. Intern Med Tokyo Jpn. 2011;50:1397-401.

67. Koo S, Baden LR, Marty FM. Post-diagnostic kinetics of the (1 $\rightarrow 3$ )- $\beta$-D-glucan assay in invasive aspergillosis, invasive candidiasis and Pneumocystis jirovecii pneumonia. Clin Microbiol Infect. 2012;18:E122-127.

68. Held J, Wagner D. $\beta$-d-Glucan kinetics for the assessment of treatment response in Pneumocystis jirovecii pneumonia. Clin Microbiol Infect. 2011;17:1118-22.

69. Wahidi MM, Lamb C, Murgu S, Musani A, Shojaee S, Sachdeva A, Maldonado F, Mahmood K, Kinsey M, Sethi S, Mahajan A, Majid A, Keyes C, Alraiyes AH, Sung A, Hsia D, Eapen G. American Association for Bronchology and Interventional Pulmonology (AABIP) Statement on the Use of Bronchoscopy and Respiratory Specimen Collection in Patients With Suspected or Confirmed COVID-19 Infection. J Bronchology Interv Pulmonol. 2020;27(4):e52-e54. https://doi.org/10.1097/LBR.0000000000 000681.

70. Chang SH, Jiang J, Kon ZN, Williams DM, Geraci TC, Smith DE, et al. Safety and efficacy of bronchoscopy in critically ill patients with coronavirus disease 2019. Chest. 2020. https://doi.org/10. 1016/j.chest.2020.09.263.

71. Gao CA, Bailey JI, Walter JM, Coleman JM, Malsin ES, Argento $\mathrm{AC}$, et al. Bronchoscopy on intubated COVID-19 patients is associated with low infectious risk to operators. Ann Am Thorac Soc. 2021. https://doi.org/10.1513/AnnalsATS.202009-1225RL.

72. Mondoni M, Sferrazza Papa GF, Rinaldo R, Faverio P, Marruchella A, D'Arcangelo F, et al. Utility and safety of bronchoscopy during the SARS-CoV-2 outbreak in Italy: a retrospective, multicentre study. Eur Respir J. 2020;56:2002767.

73. De Francesco MA, Alberici F, Bossini N, Scolari F, Pascucci F, Tomasoni G, et al. Pneumocystis jirevocii and SARS-CoV-2 CoInfection: a common feature in transplant recipients? Vaccines. 2020;8:544.

74. Menon AA, Berg DD, Brea EJ, Deutsch AJ, Kidia KK, Thurber EG, et al. A case of COVID-19 and Pneumocystis jirovecii Coinfection. Am J Respir Crit Care Med. 2020. https://doi.org/10. 1164/rccm.202003-0766LE.

75. Quintana-Ortega C, Remesal A, Ruiz de Valbuena M, de la Serna O, Laplaza-González M, Álvarez-Rojas E, et al. Fatal outcome of anti-MDA5 juvenile dermatomyositis in a paediatric COVID-19 patient: a case report. Mod Rheumatol Case Rep. 2021. https:// doi.org/10.1080/24725625.2020.1832755.

76. Bhat P, Noval M, Doub JB, Heil E. Concurrent COVID-19 and Pneumocystis jirovecii pneumonia in a severely immunocompromised 25-year-old patient. Int J Infect Dis. 2020;99:119-21.

77. Broadhurst AGB, Lalla U, Taljaard JJ, Louw EH, Koegelenberg CFN, Allwood BW. The diagnostic challenge of pneumocystis pneumonia and COVID-19 co-infection in HIV. Respirol Case Rep. 2021;9(4):e00725. Published 2021 Feb 22. https://doi.org/ $10.1002 / \mathrm{rcr} 2.725$

78. Coleman H, Snell LB, Simons R, Douthwaite ST, Lee MJ. Coronavirus disease 2019 and pneumocystis jirovecii pneumonia: a diagnostic dilemma in HIV. AIDS. 2020;34:1258-60.

79. Kelly S, Waters L, Cevik M, Collins S, Lewis J, Wu M-S, et al. Pneumocystis pneumonia, a COVID-19 mimic, reminds us of the importance of HIV testing in COVID-19. Clin Med. 2020;20:590-2. 
80. Mang S, Kaddu-Mulindwa D, Metz C, Becker A, Seiler F, Smola $\mathrm{S}$, et al. Pneumocystis jirovecii pneumonia and severe acute respiratory syndrome coronavirus 2 coinfection in a patient with newly diagnosed HIV-1 infection. Clin Infect Dis. 2020. https://doi.org/ 10.1093/cid/ciaa906.
81. Rubiano C, Tompkins K, Sellers SA, Bramson B, Eron J, Parr $\mathrm{JB}$, et al. Pneumocystis and severe acute respiratory syndrome coronavirus 2 coinfection: a case report and review of an emerging diagnostic dilemma. Open Forum Infect Dis. 2021;8:633. 